\title{
Clinical and Pathological Characteristics of Brain Tumor
}

\author{
Naziruddin Mollah ${ }^{1}$,Abdul Baki ${ }^{2}$, Nur Afzal $^{3}$, Akram Hossen ${ }^{4}$ \\ ${ }^{1}$ Resident. Department of Oncology, BSMMU. ${ }^{2}$ Senior Medical Officer, BIRDEM Hospital. ${ }^{3}$ Medical Officer, Police Hospital. ${ }^{4}$ Professor, \\ Department of Oncology, BSMMU
}

\begin{abstract}
Background: CNS tumors comprise $2 \%$ to $5 \%$ of all tumors. There was no epidemiological and statistical data regarding various features of brain tumors in this country. $80 \%$ involve the brain and $20 \%$ involve the spinal cord. Brain tumors cause approximately $2 \%$ of all cancer deaths. Objectives: to evaluate the clinical and pathological characteristics of brain tumors. Methods: This prospective study was done at Oncology department and Neurosurgery department of Bangabandhu Shiekh Mujib Medical University from July 2006 to June 2007. Total 50 patient age 2-60 years attending the oncology department with the diagnosis of primary brain tumor were included in this study. Clinical symptoms and sign in relation to brain tumor were recorded. $X$ ray skull and CT scan reports of all cases were evaluated. To find out the histopathological pattern of the brain tumors all histopathological reports were also evaluated. Results: Most common symptoms of brain tumor were headache (76\%), mental change (64\%), vomiting (52\%), visual defect (46\%), difficulty in movement (42\%) and convulsion (36\%). Astrocytoma was found in $40 \%$ patients and $30 \%$ brain tumor was associated with hydrocephalus Conclusion:This study represent the brain tumor and gives some idea about the clinicopathological aspects of the disease in our country. It will help to do further studies to evaluate the clinical, epidemiological and pathological characteristics of brain tumor.
\end{abstract}

[BSMMU J 2010; 3(2): 68-71]

\section{Introduction}

Central nervous system (CNS) tumors comprise $2 \%$ to $5 \%$ of all tumors. $80 \%$ involve the brain and $20 \%$ involve the spinal cord. Brain tumors cause approximately $2 \%$ of all cancer deaths. $60 \%$ to $80 \%$ of brains tumors are primary and rest $20 \%$ to $40 \%$ are metastatic. ${ }^{1}$ Tumors of the CNS account for as many as $20 \%$ of all cancers of childhood and are second only to leukaemia as a cause of death from malignancy. In childhood $70 \%$ of primary brain tumors are infratenorial and involve cerebellum, midbrain, pons and medulla. $^{2}$

Benign tumors of the brain tend to grow slowly and some of them may be cured by surgery with or without radiation therapy. The malignant tumors grow more rapidly and are associated with a shorter survival. Some of those highly lethal tumors, such as medulloblastoma and ependymoblastoma have a tendency to disseminate throughout the CNS.

There are increasing data documenting a genetic basis or at least a genetic association with some brain tumours. An association of brain tumours with certain chemical/drugs, Epstein-Barr virus and irradiation has been reported. ${ }^{3,4}$ In our country, there is no epidemiological and statistical data regarding various features of brain tumors. Although,

Address for Correspondence: Dr. Naziruddin Mollah, Resident. Department of Oncology, BSMMU, Dhaka the incidence of brain tumours has not been determined in our country, overall clinical experiences indicate that its incidence is not low. This study was done to evaluate the clinical and pathological characteristics of primary brain tumors.

\section{Methods:}

This retrospective study was done at Oncology department and Neurosurgery department of Bangabandhu Shiekh Mujib Medical University from July 2006 to June 2007. Total 50 patients age 2-60 years attending the oncology department with diagnosis of primary brain tumor were included in this study. Primary brain tumor was diagnosed by neurosurgery department of BSMMU.

\section{Study Procedure:}

All patients with primary brain tumor fulfilling inclusion criteria were included in the present study. A predesigned data collection sheet was used for each subject and informations regarding history, clinical examination and investigations were recorded. After inclusion of the cases, data were collected from surgeon's clinical notes. History of the patients was taken in details with special emphasis given on the following points: age, sex, religion, occupation, socioeconomic condition, educational status, residence, history of radiation exposure, family history, personal history and past history of illness with a view to 
find out any relationship with the brain tumor. Clinical symptoms and sign in relation to brain tumor were recorded. $\mathrm{X}$ ray skull and CT scan reports of all cases were evaluated. To find out the histopathological pattern of the brain tumors all histopathological reports were also evaluated.

Collected data was analyzed by using Statistical Package for Social Science (SPSS version 12.0).

\section{Results:}

Among the 50 patient $66 \%$ were male and male female ratio was 1.9:1. Mean ( \pm SD) age of the patients were $46 \pm 11$ years. Seventy percent patients came from rural area and 92\% patients were Muslim. Forty eight percent patients from middle socioeconomic background and rest from higher or lower socioeconomic status. There was no difference in socioeconomic status.

Most common symptoms of brain tumors were headache (76\%), mental change (46\%), Vomiting (52\%), visual defect (46\%), difficulty in movement (42\%) and convulsion (36\%). Others symptoms were complete blindness (20\%), dementia (14\%), unconsciousness (14\%), fever (06\%), weight gain (06\%), anorexia (04\%), vertigo (04\%), sensory loss (04\%) and loss of libido (02\%). In most of the cases more than one symptom were present (Table-I).

Table-I

Distribution of patient according to presenting symptom $(n=50)$

\begin{tabular}{lcc}
\hline Symptoms & No. of cases & Percentage \\
\hline Headache & 38 & 76.0 \\
Vomiting & 26 & 52.0 \\
Vertigo & 02 & 04.0 \\
Convulsion & 18 & 36.0 \\
Anorexia & 02 & 04.0 \\
Fever & 03 & 06.0 \\
Mental change & 32 & 64.0 \\
Dementia(past/recent memory) & 07 & 14.0 \\
Difficulty in movement & 21 & 42.0 \\
Sensory loss & 02 & 04.0 \\
Visual defect & 23 & 46.0 \\
Complete blindness & 10 & 20.0 \\
Disturbance of consciousness & 03 & 06.0 \\
Unconsciousness & 07 & 14.0 \\
Weight gain & 03 & 06.0 \\
Loss of libido & 01 & 02.0 \\
\hline
\end{tabular}

Large number of patients had signs of increased intracranial pressure (58\%), focal presentation (46\%), visual defects (50\%), and oculomotor and abducens nerve defects (12\%), other signs (less frequent) includes sensory level defects (12), facial palsy (08\%), incoordination (06\%), endocrine abnormalities (06\%), Parinaud's Syndrome (02\%). In most of the cases more than one signs were present (Table-II)

\section{Table-II}

Distribution of patient according to presenting sign $(n=50)$

\begin{tabular}{lcc}
\hline Signs & $\begin{array}{c}\text { No. of } \\
\text { Cases }\end{array}$ & Percentage \\
\hline $\begin{array}{l}\text { Focal presentation related to } \\
\text { tumor location (hemiplegia, hemi }\end{array}$ & 23 & 46.0 \\
paresis, monoplagia, aphasia) & & \\
$\begin{array}{l}\text { Sensory level defect } \\
\text { Increased intracranial pressure } \\
\text { (papilloedema) }\end{array}$ & 06 & 12.0 \\
$\begin{array}{l}\text { Visual defects } \\
\text { Oculomotor and abducens nerve } \\
\text { defects (squint/diplopia/pupillary } \\
\text { abnormality) }\end{array}$ & 16 & 58.0 \\
$\begin{array}{l}\text { Facial palsy } \\
\text { In coordination }\end{array}$ & 04 & \\
$\begin{array}{l}\text { Endocrine abnormalities } \\
\text { (Hypopituitarism/Hyperpituitarism) }\end{array}$ & 03 & 08.0 \\
Parinaud's Syndrome & 01 & 06.0 \\
\hline
\end{tabular}

$\mathrm{X}$ ray skull was done in all patients. More than $50 \%$ of case there were no radiological findings, $32 \%$ cases showed bony lesion and 14\% cases showed calcification (Fig 1). CT was done in all patients and hydrocephalus was detected in 30\% patients (Fig 2)

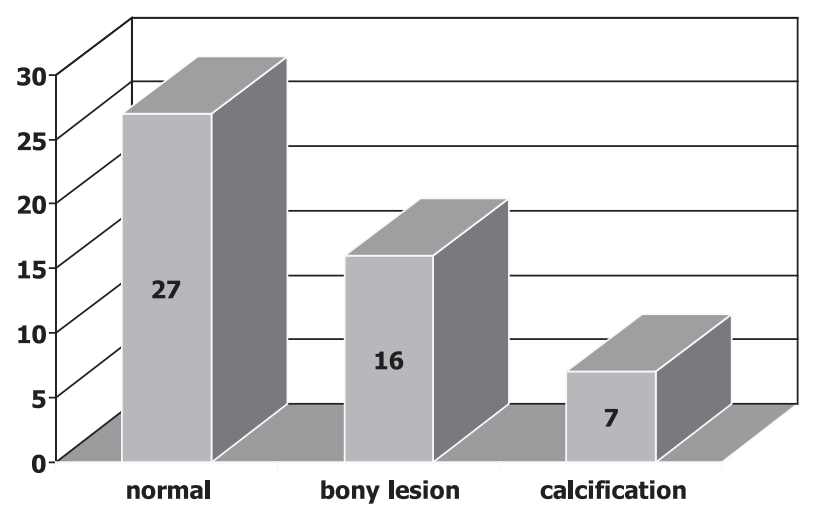

Fig.-1: Radiological finding of skull in brain tumor $(n=50)$ 


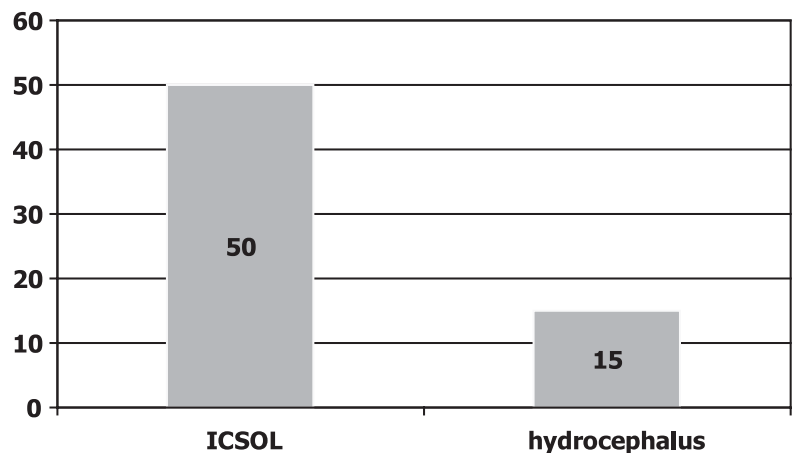

Fig.-2: CT finding of skull in brain tumor $(n=50)$

Histological examination was done in all patients. 40\% patients had astrocytomas. Pituitary tumors and Meningiomas were 16\% and 14\% respectively. Other tumor types include, Craniopharyngioma (06\%), Pineal tumour (08\%), Ependymoma (04\%), Medulloblastma (04\%), Oligodendroglioma (02\%), Brain stem tumor (02\%), Thalamic tumor (02\%) (Table-III).

Table-III

Histological finding of brain tumor $(n=50)$

\begin{tabular}{lcc}
\hline Type & No. of cases & Percentage \\
\hline Astrocytoma & 20 & 40.0 \\
$\quad$ Grade-I & 06 & 12.0 \\
$\quad$ Grade-II & 09 & 18.0 \\
Grade-III & 02 & 04.0 \\
$\quad$ Grade-IV & 03 & 06.0 \\
Meningioma & 07 & 14.0 \\
Pituitary tumour & 08 & 16.0 \\
Craniopharyngioma & 03 & 06.0 \\
Pineal tumour & 04 & 08.0 \\
Ependymoma & 02 & 04.0 \\
Medulloblastma & 02 & 04.0 \\
Oligodendroglioma & 01 & 02.0 \\
Brain stem tumour (radiologically) & 01 & 02.0 \\
Brain tumour & 01 & 02.0 \\
(Thalamic radiologically) & & \\
\hline
\end{tabular}

\section{Discussion:}

The present study is a clinicopathological study of brain tumor. Clinical presentation may very according the site and type of brain tumor, although there are some common symptoms, namely, headache, seizure, mental change and sensorimotor defects and any of which can eventually be found in more than $50 \%$ of the patient population. Ocular change (e.g. visual defect) is associated with the tumors, like, pituitary adenoma craniopharyngioma optic nerve glioma and pineal growth. Most of the tumors present focal presentation related to tumors location. ${ }^{5}$ In this series, $76 \%$ of case present headache, $64 \%$ mental changes, $52 \%$ vomiting, 46\% visual defect, 36\% convulsion, 20\% completely blind.

Physical findings can be variable according to tumor type and location. Common signs are focal presentation related to location of tumor, and increased intracranial pressure (ICP) and abducence and oculomotor nerve defects,. Ocular changes are accompaniment of optic glioma pituitary adenoma and pineal growth. Endocrine abnormalities may be found in case of pituitary adenoma and pineal tumor. ${ }^{5}$ In the present series, 58\% cases had ICP, 50\% visual defects, $46 \%$ focal presentation related to tumor location and $32 \%$ cases abducence and oculomotor nerve defects.

In this study skull radiography and CT scan were done in all patients. Skull radiography is poor in detecting brain tumour. Intracranial space occupying lesion (ICSOL) was found in all patients. Some degree of hydrocephalus was associated with 30\% patients. Biopsy and histopathology were also performed in all patients. In this study astrocytomas were found in $40 \%$ of cases, among them $22 \%$ are grade II, $10 \%$ grade I, $6 \%$ grade III and $4 \%$ grade IV (glioblastoma multiforme). Pituitary tumors were $16 \%$ pineal tumor 6\% ependymoma, 4\% medulloblastoma 4\%, oligodendroglioma $2 \%$ and brain stem tumor $2 \%$. Allen and Chutorian found gliomas $50 \%$ of all primary brain tumors and glioblastomas comprise over $50 \%$ of all glioma, meningioma constitutes $15 \%$ of primary intracranial neoplasm. Pituitary adenomas comprise $12 \%$ to $18 \%$ of intracranial neoplasm, the majority of which are chromo phobic; they are almost never malignant. ${ }^{3,6}$ Walker MD found medulloblastoma represents $4 \%$ to $8 \%$ of all primary brain tumor and ependymomas account for $1 \%$ to $8 \% .{ }^{4}$ Percentage of gliomas was correlates with the study done by Allen and Chutorian but the grading of the tumor markedly varies and almost reverse. In the previous study it was found that more than $50 \%$ of gliomas are in advanced grade, ${ }^{3}$ but in this study maximum glioma cases are found in grade I. \& II Percentage of pituitary adenoma, meningioma and other tumors well correlate with the previous study.

In conclusion in this study most common manifestation of brain tumor was vomiting, convulsion, headache, visual defect, papilloedema, focal neurological sign, oculomotor and abducens nerve defects. One third of the patient had associated hydrocephalus. Astrocytoma was the common tumor of brain followed by Meningioma and Pineal tumour. 
Diagnostic yield of x-ray skull is poor. MRI or CT scan should be done in any suspected case of Brain tumour.

\section{References:}

1. Rubin P, Mc Donald S, Qazi R. Clinical oncology, 7th edition. Philadelphia: WE saunders ; 1993.

2. Burger PC, Scheithauer BW, Vogel FS. Surgical pathology of nervous system and its covering, $3^{\text {rd }}$ edition. New York : Churehill Livingstone; 1991.

3. Allen JC. Childhood brain tumors: Current status of clinical trials in newly diagnosed and recurrent disease. Clin N Am. 19961 32: 633-51.
4. Walker MD. Brain and peripheral nervous system tumors. In: Holland JF, Feri E (eds). Cancer medicine. Philadelphia : Lea \& Febiger; 1993, pp 1385-1417.

5. Kornbilth PL. Increased intracranial pressure. In: Devita VT, Hellmann S, Rosenberg SA (eds): Cancer: principles and practice of oncology. Philadelphia : JB Lippincott; 1992, p. 15861588.

6. Chutorian AM, Grati SR: Diagnosis of intracranial tumors in infants and children. In: Chang $\mathrm{CH}$, Houspian EM (eds): Tumors of the Central Nervous system: Modern Radiotherapy in Multidisciplinary Management New York, Masson publishing 1992, pp 83-125. 